\title{
SLICING INEQUALITIES FOR SUBSPACES OF $L_{p}$
}

\author{
ALEXANDER KOLDOBSKY
}

(Communicated by Thomas Schlumprecht)

ABSTRACT. We prove slicing inequalities for measures of the unit balls of subspaces of $L_{p},-\infty<p<\infty$. For example, for every $k \in \mathbb{N}$ there exists a constant $C(k)$ such that for every $n \in \mathbb{N}, k<n$, every convex $k$-intersection body (unit ball of a normed subspace of $L_{-k}$ ) $L$ in $\mathbb{R}^{n}$ and every measure $\mu$ with non-negative even continuous density in $\mathbb{R}^{n}$,

$$
\mu(L) \leq C(k) \max _{\xi \in S^{n-1}} \mu\left(L \cap \xi^{\perp}\right)|L|^{1 / n},
$$

where $\xi^{\perp}$ is the central hyperplane in $\mathbb{R}^{n}$ perpendicular to $\xi$, and $|L|$ is the volume of $L$. This and other results are in the spirit of the hyperplane problem of Bourgain. The proofs are based on stability inequalities for intersection bodies and estimates for the Banach-Mazur distance from the unit ball of a subspace of $L_{p}$ to the class of intersection bodies.

\section{INTRODUCTION}

The hyperplane problem [1,3,4,31] asks whether there exists an absolute constant $C$ so that for any origin-symmetric convex body $K$ in $\mathbb{R}^{n}$

$$
|K|^{\frac{n-1}{n}} \leq C \max _{\xi \in S^{n-1}}\left|K \cap \xi^{\perp}\right|,
$$

where $\xi^{\perp}$ is the central hyperplane in $\mathbb{R}^{n}$ perpendicular to $\xi$, and $|K|$ stands for volume of proper dimension. The problem is still open. Bourgain [5] proved that $C \leq O\left(n^{1 / 4} \log n\right)$, and Klartag [13] removed the logarithmic term and established the best-to-date estimate $C \leq O\left(n^{1 / 4}\right)$. We refer the reader to [6] for the history and partial results.

Inequality (1) has been proved for several special classes of bodies. For instance, if $K$ is an intersection body, the inequality is known with the best possible constant (see [7, Th. 9.4.11]):

$$
|K|^{\frac{n-1}{n}} \leq c_{n} \max _{\xi \in S^{n-1}}\left|K \cap \xi^{\perp}\right|,
$$

where $c_{n}=\left|B_{2}^{n}\right|^{\frac{n-1}{n}} /\left|B_{2}^{n-1}\right|<1$, and $B_{2}^{n}$ is the unit Euclidean ball. One possible path to attack the hyperplane problem is through the scale of finite dimensional subspaces of $L_{p}$, because one approximately gets all origin-symmetric convex bodies, as $p \rightarrow+\infty$. It was shown by Ball [2] that the hyperplane problem has an affirmative answer for the unit balls of finite dimensional subspaces of $L_{p}, 1 \leq p \leq 2$. Junge [9] extended this result to every $p \in(1, \infty)$, with the constant $C$ depending on $p$ and

Received by the editors July 20, 2014 and, in revised form, December 26, 2014.

2010 Mathematics Subject Classification. Primary 52A20; Secondary 46B07.

This work was partially supported by the US National Science Foundation, grant DMS1265155 . 
going to infinity when $p \rightarrow \infty$. Milman 28 gave a different proof for subspaces of $L_{p}, 2 \leq p<\infty$, with the constant $C \leq O(\sqrt{p})$. Another proof of this estimate can be found in 23 .

The paper [23] also explored a different path, through $n$-dimensional spaces that embed in $L_{-p}, 0<p<n$. The latter concept was introduced in 15, 17; see definition and basic facts below. In particular, a normed space embeds in $L_{-k}$ if and only if its unit ball belongs to the class of $k$-intersection bodies introduced in [16, 17] in connection with generalizations of the Busemann-Petty problem. The path through $L_{-p}$ presents some hope because every $n$-dimensional normed space embeds in $L_{-p}$ with $n-3 \leq p<n$. However, the paper [23] did not go very far, as the hyperplane conjecture was confirmed there only for the unit balls of spaces that embed in $L_{-p}, 0<p<2$, with the constant going to infinity as $p \rightarrow-2$.

In this note we prove the hyperplane conjecture for the unit balls of normed spaces that embed in $L_{-p}$ for arbitrary $p>0$, and, in particular, for $k$-intersection bodies for all $k \in \mathbb{N}$. Moreover, we prove the result with arbitrary measures in place of volume.

The first result on the hyperplane problem for arbitrary measures was proved in [19]. If $K$ is an intersection body in $\mathbb{R}^{n}$ and $\mu$ is any measure with even continuous density $f$ in $\mathbb{R}^{n}$ (so that $\mu(B)=\int_{B} f$ for every compact set $B \subset \mathbb{R}^{n}$ ), then

$$
\mu(K) \leq \frac{n}{n-1} c_{n} \max _{\xi \in S^{n-1}} \mu\left(K \cap \xi^{\perp}\right)|K|^{1 / n},
$$

where $c_{n}$ is the same constant as in (2). The constant in (3) is sharp and differs from the case of volume in (2) by a small factor of $n /(n-1)$. Recall that $c_{n}<1$.

The condition that $K$ is an intersection body in inequality (3) was removed in [20] at the expense of extra $\sqrt{n}$ in the right-hand side. For any origin-symmetric convex body $K$ in $\mathbb{R}^{n}$ and any measure $\mu$ with even continuous density in $\mathbb{R}^{n}$,

$$
\mu(K) \leq \sqrt{n} \frac{n}{n-1} c_{n} \max _{\xi \in S^{n-1}} \mu\left(K \cap \xi^{\perp}\right)|K|^{1 / n} .
$$

Analogs of (44) for sections of lower dimensions and for complex convex bodies were proved in 21.

Our main result is the following inequality. For any $p>0$, there exists a constant $C(p)$, such that for any $n \in \mathbb{N}, n>p$, any convex body $L$ in $\mathbb{R}^{n}$ that is the unit ball of a normed space embedding in $L_{-p}$, any $1 \leq m \leq n-1$, and any measure $\mu$ with even continuous non-negative density in $\mathbb{R}^{n}$,

$$
\mu(L) \leq(C(p))^{m} \frac{n}{n-m} \max _{H \in G r_{n-m}} \mu(L \cap H)|L|^{m / n},
$$

where $G r_{n-m}$ is the Grassmanian of $(n-m)$-dimensional subspaces of $\mathbb{R}^{n}$. In the case where $p=k \in \mathbb{N}$, inequality (5) applies to any convex $k$-intersection body $L$.

We also show that the constant $\sqrt{n}$ in (4) can be replaced by $n^{1 / 2-1 / p}$ when $K$ is the unit ball of an $n$-dimensional subspace of $L_{p}, p>2$. The proofs are based on a stability result for intersection bodies, which was proved in [21] and continued the study of stability in volume comparison problems initiated in [18]; see also [22].

\section{Embedding in $L_{-p}$ AND $k$-INTERSECTION BOdieS}

We need several definitions and facts. A closed bounded set $K$ in $\mathbb{R}^{n}$ is called a star body if every straight line passing through the origin crosses the boundary of 
$K$ at exactly two points different from the origin, the origin is an interior point of $K$, and the Minkowski functional of $K$ defined by

$$
\|x\|_{K}=\min \{a \geq 0: x \in a K\}
$$

is a continuous function on $\mathbb{R}^{n}$.

The radial function of a star body $K$ is defined by

$$
\rho_{K}(x)=\|x\|_{K}^{-1}, \quad x \in \mathbb{R}^{n}, x \neq 0 .
$$

If $x \in S^{n-1}$, then $\rho_{K}(x)$ is the radius of $K$ in the direction of $x$.

The class of intersection bodies was introduced by Lutwak 26. Let $K, L$ be origin-symmetric star bodies in $\mathbb{R}^{n}$. We say that $K$ is the intersection body of $L$ if the radius of $K$ in every direction is equal to the $(n-1)$-dimensional volume of the section of $L$ by the central hyperplane orthogonal to this direction, i.e., for every $\xi \in S^{n-1}$,

$$
\rho_{K}(\xi)=\|\xi\|_{K}^{-1}=\left|L \cap \xi^{\perp}\right| .
$$

All bodies $K$ that appear as intersection bodies of different star bodies form the class of intersection bodies of star bodies. The class of intersection bodies can be defined as the closure of the class of intersection bodies of star bodies in the radial metric

$$
\rho(K, L)=\sup _{\xi \in S^{n-1}}\left|\rho_{K}(\xi)-\rho_{L}(\xi)\right| .
$$

A more general concept of a $k$-intersection body was introduced in 16, 17. For an integer $k, 1 \leq k<n$ and star bodies $D, L$ in $\mathbb{R}^{n}$, we say that $D$ is the $k$-intersection body of $L$ if for every $(n-k)$-dimensional subspace $H$ of $\mathbb{R}^{n}$,

$$
\left|D \cap H^{\perp}\right|=|L \cap H|,
$$

where $H^{\perp}$ is the $k$-dimensional subspace orthogonal to $H$. Taking the closure in the radial metric of the class of all $D$ 's that appear as $k$-intersection bodies of star bodies, we define the class of $k$-intersection bodies (the original definition in [16, 17. was different; the equivalence of definitions was proved by Milman [29]). If $k=1$, one gets the usual intersection bodies. Intersection bodies played a crucial role in the solution of the Busemann-Petty problem and its generalizations; see 14, Ch. 5].

Another generalization of intersection bodies was introduced by Zhang [36] in connection with the lower dimensional Busemann-Petty problem. For $1 \leq m \leq$ $n-1$, denote by $G r_{n-m}$ the Grassmanian of $(n-m)$-dimensional subspaces of $\mathbb{R}^{n}$. The $(n-m)$-dimensional spherical Radon transform $R_{n-m}: C\left(S^{n-1}\right) \mapsto C\left(G r_{n-m}\right)$ is a linear operator defined by

$$
R_{n-m} g(H)=\int_{S^{n-1} \cap H} g(x) d x, \quad \forall H \in G r_{n-m}
$$

for every function $g \in C\left(S^{n-1}\right)$. Denote by

$$
R_{n-m}\left(C\left(S^{n-1}\right)\right)=X \subset C\left(G r_{n-m}\right) .
$$

Let $M^{+}(X)$ be the space of linear positive continuous functionals on $X$, i.e., for every $\nu \in M^{+}(X)$ and non-negative function $f \in X$, we have $\nu(f) \geq 0$. 
An origin-symmetric star body $K$ in $\mathbb{R}^{n}$ is called a generalized $m$-intersection body if there exists a functional $\nu \in M^{+}(X)$, so that for every $g \in C\left(S^{n-1}\right)$,

$$
\int_{S^{n-1}}\|x\|_{K}^{-m} g(x) d x=\nu\left(R_{n-m} g\right) \text {. }
$$

It was shown in [17] (see [14, p. 92] or 29] for different proofs) that every generalized $m$-intersection body is an $m$-intersection body (it sounds counterintuitive, but the class of $m$-intersection bodies is larger). Note that generalized 1-intersection bodies coincide with the original intersection bodies of Lutwak. We denote the class of generalized $m$-intersection bodies by $B P_{m}$.

The concept of embedding of finite dimensional normed spaces in $L_{-p}$ with $p>0$ was introduced in [15, 17] as an analytic extension of embedding of normed spaces into $L_{p}$ with $p>0$. It is a well-known fact going back to P. Levy (see for example [14, Lemma 6.4]) that an $n$-dimensional normed space $\left(\mathbb{R}^{n},\|\cdot\|\right)$ embeds in $L_{p}([0,1]), p>-1$ if and only if there exists a finite Borel measure $\mu$ on the sphere $S^{n-1}$ in $\mathbb{R}^{n}$ so that for every $x \in \mathbb{R}^{n}$,

$$
\|x\|^{p}=\int_{S^{n-1}}|(x, \xi)|^{p} d \mu(\xi) .
$$

We say that a normed space embeds in $L_{p}$ if its norm satisfies (8), without associating $L_{p}$ with a measure space. Embedding in $L_{-p}$ is defined as an analytic extension of (8).

Definition 1. Let $X$ be an $n$-dimensional normed space, and $0<p<n$. We say that $X$ embeds in $L_{-p}$ if there exists a finite Borel measure $\mu$ on $S^{n-1}$ so that, for every even Schwartz test function $\phi \in \mathcal{S}\left(\mathbb{R}^{n}\right)$,

$$
\int_{\mathbb{R}^{n}}\|x\|_{X}^{-p} \phi(x) d x=\int_{S^{n-1}}\left(\int_{\mathbb{R}}|t|^{p-1} \hat{\phi}(t \theta) d t\right) d \mu(\theta)
$$

where $\hat{\phi}$ stands for the Fourier transform.

A connection between intersection bodies and embedding in $L_{p}$ was established in [17, Th. 3].

Proposition 1. Let $1 \leq k<n$. The following are equivalent:

(i) An origin symmetric star body $D$ in $\mathbb{R}^{n}$ is a $k$-intersection body;

(ii) $\|\cdot\|_{D}^{-k}$ represents a positive definite distribution;

(iii) the space $\left(\mathbb{R}^{n},\|\cdot\|_{D}\right)$ embeds in $L_{-k}$.

The advantage of this connection (and, consequently, of introducing embeddings in $L_{-p}$ ) is that now one can try to extend to negative values of $p$ different results about usual $L_{p}$-spaces. Every such extension gives new information about intersection bodies. Let us show several examples of this approach.

A well-known simple fact is that every two-dimensional normed space embeds in $L_{1}$. It can be proved (see 14, Theorem 4.13]) that for every symmetric convex body $K$ in $\mathbb{R}^{n}$ and every $p \in[n-3, n)$, the function $\|\cdot\|_{K}^{-p}$ represents a positive definite distribution, so by Proposition 1 every $n$-dimensional Banach space embeds in $L_{-n+3}$. Putting $n=2$ we get the property of two-dimensional spaces mentioned above. Putting $n=4$ we see that every four-dimensional normed space embeds in $L_{-1}$. By Proposition 1, every four-dimensional symmetric convex body is a 1intersection body, which solves in the affirmative the critical four-dimensional case of the Busemann-Petty problem; see [14, Ch. 5]. 
Another well-known property of $L_{p}$-spaces is that, for any $0<p<q \leq 2$, the space $L_{q}$ embeds isometrically in $L_{p}$ (see for example [14, Corollary 6.7]), so $L_{p^{-}}$ spaces become larger when $p$ decreases from 2 . This result can be extended to negative $p$ as follows.

Proposition 2 ([14, Th. 6.17]). Every $n$-dimensional subspace of $L_{q}, 0<q \leq 2$ embeds in $L_{-p}$ for every $p \in(0, n)$. Hence, the unit ball of every $n$-dimensional subspace of $L_{q}, 0<q \leq 2$ is a $k$-intersection body for every $1 \leq k<n$.

The factorization theorem of Maurey and Nikišin [27, 32] implies that, for $0<$ $p<q<1$, every Banach subspace of $L_{p}$ is isomorphic to a subspace of $L_{q}$. The following extension to negative $p$ was proved in [10] (see also [14, Section 6.3]). Recall that the geometric distance between two star bodies $K$ and $L, d_{G}(K, L)$, is defined as the infimum of positive numbers $r$ such that there exists some $a>0$ so that

$$
K \subset a L \subset r K
$$

and the Banach-Mazur distance is defined by

$$
d_{B M}(K, L)=\inf _{T \in G L_{n}} d_{G}(K, T L) .
$$

Proposition $3([10])$. If $-\infty<p<q<1, p \neq 0$ and $q>0$, then there exists a constant $C=C(p, q)$ so that, for any $n$ with $-n<p$, if $X$ is an $n$-dimensional normed space that embeds into $L_{p}$, then there is a subspace $Y$ of $L_{q}$ so that $d_{B M}(K, L) \leq C$, where $K$ and $L$ are the unit balls of $X$ and $Y$, respectively.

We refer the reader to [12, 14, 24, 29, 30, 34, 36] for more about $k$-intersection bodies, embedding in $L_{-p}$ and their applications.

\section{SLicing INEQUALities}

We use the following stability result for generalized intersection bodies proved in $[21$.

Proposition 4 ([21]). Suppose that $1 \leq m \leq n-1, K$ is a generalized $m$ intersection body in $\mathbb{R}^{n}, f$ is an even continuous function on $K, f \geq 1$ everywhere on $K$, and $\varepsilon>0$. If

$$
\int_{K \cap H} f \leq|K \cap H|+\varepsilon, \quad \forall H \in G r_{n-m}
$$

then

$$
\int_{K} f \leq|K|+\frac{n}{n-m} c_{n, m}|K|^{m / n} \varepsilon,
$$

where $c_{n, m}=\left|B_{2}^{n}\right|^{\frac{n-m}{n}} /\left|B_{2}^{n-m}\right|$; note that $c_{n, m} \in\left(\left(\frac{1}{e}\right)^{m / 2}, 1\right)$ and that $c_{n, 1}$ equals the constant $c_{n}$ from (2).

We define the Banach-Mazur distance from a star body $L$ in $\mathbb{R}^{n}$ to the class $B P_{m}$ of generalized $m$-intersection bodies by

$$
d_{B M}\left(L, B P_{m}\right)=\inf _{K \in B P_{m}} d_{B M}(L, K) .
$$


Theorem 1. Suppose that $1 \leq m \leq n-1$ and $L$ is a star body in $\mathbb{R}^{n}$ such that $d_{B M}\left(L, B P_{m}\right)<d$ for some $d>0$. Then for any measure $\mu$ with even continuous non-negative density on $L$

$$
\mu(L) \leq d^{m} \frac{n}{n-m} c_{n, m} \max _{H \in G r_{n-m}} \mu(L \cap H)|L|^{m / n} .
$$

Proof. Since any linear transformation of a generalized $m$-intersection body is again a generalized $m$-intersection body (see [8, Corollary 6.8]), there exists $K \in B P_{m}$ such that

$$
\frac{1}{d} K \subset L \subset K, \quad \text { and then } \quad \frac{1}{d} K \subset-L \subset K .
$$

Let $g$ be the density of $\mu$, and let $f=\chi_{K}+(1 / 2) g\left(\chi_{L}+\chi_{-L}\right)$, where $\chi_{K}, \chi_{L}, \chi_{-L}$ are the indicator functions of $K, L$ and $-L$. Then $f \geq 1$ everywhere on $K$. Put

$$
\varepsilon=\max _{H \in G r_{n-m}}\left(\int_{K \cap H} f-|K \cap H|\right)=\max _{H \in G r_{n-m}} \int_{L \cap H} g .
$$

Now we can apply Proposition 4 to $f, K, \varepsilon$ (the function $f$ is not necessarily continuous on $K$, but the result holds by a simple approximation argument). We get

$$
\begin{gathered}
\mu(L)=\int_{L} g=\int_{K} f-|K| \\
\leq \frac{n}{n-m} c_{n, m}|K|^{m / n} \max _{H \in G r_{n-m}} \int_{L \cap H} g \\
\leq d^{m} \frac{n}{n-m} c_{n, m}|L|^{m / n} \max _{H \in G r_{n-m}} \mu(L \cap H),
\end{gathered}
$$

because $K \subset d L$, so $|K| \leq d^{n}|L|$.

Combining Theorem 11 with Proposition 3. we prove a slicing inequality for subspaces of $L_{-p}$.

Theorem 2. For any $p>0$ there exists a constant $C(p)$ such that for any $n \in$ $\mathbb{N}, n>p$, any convex body $L$ in $\mathbb{R}^{n}$ that is the unit ball of a normed space embedding in $L_{-p}$, any $1 \leq m \leq n-1$, and any measure $\mu$ with even continuous non-negative density in $\mathbb{R}^{n}$,

$$
\mu(L) \leq(C(p))^{m} \frac{n}{n-m} c_{n, m} \max _{H \in G r_{n-m}} \mu(L \cap H)|L|^{m / n} .
$$

Recall that $c_{n, m}<1$.

Proof. By Proposition 3, there exists a body $K$ which is the unit ball of a normed space that embeds in $L_{1 / 2}$ such that $d_{B M}(K, L)<C(p, 1 / 2)=: C(p)$. By Proposition 2, $K$ is an intersection body. By [8, Lemma 6.1], every intersection body is a generalized $m$-intersection body for every $1 \leq m \leq n-1$, so $K \in B P_{m}$. The result follows from Theorem 1 ,

The author is unable to estimate the behavior of the constant $C(p)$ as $p \rightarrow \infty$, because the proof of Proposition 3 is rather involved. For $p=k \in \mathbb{N}$, Theorem 2 can be reformulated using Proposition 1 
Corollary 1. For any $k \in \mathbb{N}$ there exists a constant $C(k)$ such that for any $n \in$ $\mathbb{N}, n>k$, any convex $k$-intersection body $L$ in $\mathbb{R}^{n}$, any $1 \leq m \leq n-1$, and any measure $\mu$ with even continuous density in $\mathbb{R}^{n}$,

$$
\mu(L) \leq(C(k))^{m} \frac{n}{n-m} c_{n, m} \max _{H \in G r_{n-m}} \mu(L \cap H)|L|^{m / n} .
$$

Let us formulate the hyperplane cases $(m=1)$ of Theorem 2 and Corollary 1 separately, as they relate to the hyperplane problem.

Corollary 2. For any $p>0$ there exists a constant $D(p)$ such that for any $n \in$ $\mathbb{N}, n>p$, any convex body $L$ in $\mathbb{R}^{n}$ that is the unit ball of a normed space embedding in $L_{-p}$ and any measure $\mu$ with even continuous non-negative density in $\mathbb{R}^{n}$,

$$
\mu(L) \leq D(p) \max _{\xi \in S^{n-1}} \mu\left(L \cap \xi^{\perp}\right)|L|^{1 / n} .
$$

When $p=k \in \mathbb{N}, 1 \leq k \leq n-1$ the latter inequality applies to any convex $k$-intersection body $L$ in $\mathbb{R}^{n}$; see the formulation in the abstract.

Finally, let us prove a slicing inequality for subspaces of $L_{p}, p>2$ with arbitrary measures.

Theorem 3. Suppose that $p>2, L$ is the unit ball of an $n$-dimensional subspace of $L_{p}, 1 \leq m \leq n-1$, and $\mu$ is a measure with even continuous density in $\mathbb{R}^{n}$. Then

$$
\mu(L) \leq n^{m / 2-m / p} \frac{n}{n-m} c_{n, m} \max _{H \in G r_{n-m}} \mu(L \cap H)|L|^{m / n} .
$$

Proof. By a result of Lewis 25] (see also 33 for a different proof), $d_{B M}\left(L, B_{2}^{n}\right) \leq$ $n^{1 / 2-1 / p}$. Since $B_{2}^{n} \in B P_{m}$ for every $m$, the result follows from Theorem 1 .

The hyperplane case is as follows.

Corollary 3. If $L$ is the unit ball of an $n$-dimensional subspace of $L_{p}, p>2$, then for any measure $\mu$ with even continuous density in $\mathbb{R}^{n}$,

$$
\mu(L) \leq n^{1 / 2-1 / p} \frac{n}{n-1} c_{n} \max _{\xi \in S^{n-1}} \mu\left(L \cap \xi^{\perp}\right)|L|^{1 / n} .
$$

Remark. In Theorems 2 and 3 we consider $L_{p}$-spaces with $p<0$ and $p>2$. As follows from Proposition 2 the unit balls of subspaces of $L_{p}$ with $0<p \leq 2$ are intersection bodies and satisfy inequalities (2) and (3). In the remaining case $p=0$, the concept of embedding in $L_{0}$ was introduced and studied in 11. As proved in [11, Th. 6.3], the unit balls of spaces that embed in $L_{0}$ are intersection bodies and also satisfy inequalities (2) and (3).

\section{REFERENCES}

[1] K. Ball, Isometric problems in $\ell_{p}$ and sections of convex sets, Ph.D. dissertation, Trinity College, Cambridge (1986).

[2] Keith Ball, Normed spaces with a weak-Gordon-Lewis property, Functional analysis (Austin, TX, 1987/1989), Lecture Notes in Math., vol. 1470, Springer, Berlin, 1991, pp. 36-47, DOI 10.1007/BFb0090210. MR.1126735 (93e:46013)

[3] J. Bourgain, On high-dimensional maximal functions associated to convex bodies, Amer. J. Math. 108 (1986), no. 6, 1467-1476, DOI 10.2307/2374532. MR868898 (88h:42020)

[4] J. Bourgain, Geometry of Banach spaces and harmonic analysis, Proceedings of the International Congress of Mathematicians, Vol. 1, 2 (Berkeley, Calif., 1986), Amer. Math. Soc., Providence, RI, 1987, pp. 871-878. MR.934289 (89d:42001) 
[5] J. Bourgain, On the distribution of polynomials on high-dimensional convex sets, Geometric aspects of functional analysis (1989-90), Lecture Notes in Math., vol. 1469, Springer, Berlin, 1991, pp. 127-137, DOI 10.1007/BFb0089219. MR1122617 (92j:52007)

[6] S. Brazitikos, A. Giannopoulos, P. Valettas, and B. Vritsiou, Geometry of isotropic logconcave measures, preprint.

[7] Richard J. Gardner, Geometric tomography, 2nd ed., Encyclopedia of Mathematics and its Applications, vol. 58, Cambridge University Press, Cambridge, 2006. MR 2251886 (2007i:52010)

[8] Eric Grinberg and Gaoyong Zhang, Convolutions, transforms, and convex bodies, Proc. London Math. Soc. (3) 78 (1999), no. 1, 77-115, DOI 10.1112/S0024611599001653. MR1658156 (99m:52009)

[9] Marius Junge, Hyperplane conjecture for quotient spaces of $L_{p}$, Forum Math. 6 (1994), no. 5, 617-635, DOI 10.1515/form.1994.6.617. MR1295155 (95g:46026)

[10] N. J. Kalton and A. Koldobsky, Intersection bodies and $L_{p}$-spaces, Adv. Math. 196 (2005), no. 2, 257-275, DOI 10.1016/j.aim.2004.09.002. MR2166308(2006d:46013)

[11] N. J. Kalton, A. Koldobsky, V. Yaskin, and M. Yaskina, The geometry of $L_{0}$, Canad. J. Math. 59 (2007), no. 5, 1029-1049, DOI 10.4153/CJM-2007-044-0. MR2354401 (2008j:52007)

[12] N. J. Kalton and M. Zymonopoulou, Positive definite distributions and normed spaces, Adv. Math. 227 (2011), no. 2, 986-1018, DOI 10.1016/j.aim.2011.02.019. MR2793030 (2012g:52009)

[13] B. Klartag, On convex perturbations with a bounded isotropic constant, Geom. Funct. Anal. 16 (2006), no. 6, 1274-1290, DOI 10.1007/s00039-006-0588-1. MR2276540 (2007i:52005)

[14] Alexander Koldobsky, Fourier analysis in convex geometry, Mathematical Surveys and Monographs, vol. 116, American Mathematical Society, Providence, RI, 2005. MR2132704 (2006a:42007)

[15] Alexander Koldobsky, Positive definite distributions and subspaces of $L_{-p}$ with applications to stable processes, Canad. Math. Bull. 42 (1999), no. 3, 344-353, DOI 10.4153/CMB-1999040-5. MR1703694 (2001i:42014)

[16] A. Koldobsky, A generalization of the Busemann-Petty problem on sections of convex bodies, Israel J. Math. 110 (1999), 75-91, DOI 10.1007/BF02808176. MR1750442 (2000m:52017)

[17] A. Koldobsky, A functional analytic approach to intersection bodies, Geom. Funct. Anal. 10 (2000), no. 6, 1507-1526, DOI 10.1007/PL00001659. MR1810751 (2001m:52007)

[18] Alexander Koldobsky, Stability in the Busemann-Petty and Shephard problems, Adv. Math. 228 (2011), no. 4, 2145-2161, DOI 10.1016/j.aim.2011.06.031. MR2836117(2012h:52019)

[19] Alexander Koldobsky, A hyperplane inequality for measures of convex bodies in $\mathbb{R}^{n}, n \leq$ 4, Discrete Comput. Geom. 47 (2012), no. 3, 538-547, DOI 10.1007/s00454-011-9362-8. MR2891246(2012m:52013)

[20] Alexander Koldobsky, $A \sqrt{n}$ estimate for measures of hyperplane sections of convex bodies, Adv. Math. 254 (2014), 33-40, DOI 10.1016/j.aim.2013.12.029. MR3161089

[21] A. Koldobsky, Estimates for measures of sections of convex bodies, arXiv:1309.6485

[22] A. Koldobsky, Stability and separation in volume comparison problems, Math. Model. Nat. Phenom. 8 (2013), no. 1, 156-169, DOI 10.1051/mmnp/20138111. MR3022986

[23] A. Koldobsky, A. Pajor, and V. Yaskin, Inequalities of the Kahane-Khinchin type and sections of $L_{p}$-balls, Studia Math. 184 (2008), no. 3, 217-231, DOI 10.4064/sm184-3-2. MR2369140 (2008m:52006)

[24] Alexander Koldobsky and Vladyslav Yaskin, The interface between convex geometry and harmonic analysis, CBMS Regional Conference Series in Mathematics, vol. 108, Published for the Conference Board of the Mathematical Sciences, Washington, DC; by the American Mathematical Society, Providence, RI, 2008. MR2365157(2008j:52008)

[25] D. R. Lewis, Finite dimensional subspaces of $L_{p}$, Studia Math. 63 (1978), no. 2, 207-212. MR.511305 (80b:46043)

[26] Erwin Lutwak, Intersection bodies and dual mixed volumes, Adv. in Math. 71 (1988), no. 2, 232-261, DOI 10.1016/0001-8708(88)90077-1. MR963487(90a:52023)

[27] B. Maurey, Théoremes de factorization pour les operatèurs linéaires à valeurs dans les espaces $L_{p}$. Astérisque 11 (1974), Société Math. de France.

[28] Emanuel Milman, Dual mixed volumes and the slicing problem, Adv. Math. 207 (2006), no. 2, 566-598, DOI 10.1016/j.aim.2005.09.008. MR2271017(2007i:52006) 
[29] Emanuel Milman, Generalized intersection bodies, J. Funct. Anal. 240 (2006), no. 2, 530-567, DOI 10.1016/j.jfa.2006.04.004. MR2261694(2007h:52007)

[30] Emanuel Milman, Generalized intersection bodies are not equivalent, Adv. Math. 217 (2008), no. 6, 2822-2840, DOI 10.1016/j.aim.2007.11.007. MR.2397468(2009k:52012)

[31] V. D. Milman and A. Pajor, Isotropic position and inertia ellipsoids and zonoids of the unit ball of a normed $n$-dimensional space, Geometric aspects of functional analysis (1987-88), Lecture Notes in Math., vol. 1376, Springer, Berlin, 1989, pp. 64-104, DOI 10.1007/BFb0090049. MR:1008717 (90g:52003)

[32] E. M. Nikišin, Resonance theorems and superlinear operators (Russian), Uspehi Mat. Nauk 25 (1970), no. 6(156), 129-191. MR0296584 (45 \#5643)

[33] Gideon Schechtman and Artem Zvavitch, Embedding subspaces of $L_{p}$ into $l_{p}^{N}, 0<p<$ 1, Math. Nachr. 227 (2001), 133-142, DOI 10.1002/1522-2616(200107)227:1/133::AIDMANA133〉3.3.CO;2-\#. MR1840560 (2002f:46042)

[34] Vladyslav Yaskin, On strict inclusions in hierarchies of convex bodies, Proc. Amer. Math. Soc. 136 (2008), no. 9, 3281-3291, DOI 10.1090/S0002-9939-08-09424-0. MR2407094 (2009h:52010)

[35] Vladyslav Yaskin, Counterexamples to convexity of $k$-intersection bodies, Proc. Amer. Math. Soc. 142 (2014), no. 12, 4355-4363, DOI 10.1090/S0002-9939-2014-12254-4. MR3267003

[36] Gaoyong Zhang, Sections of convex bodies, Amer. J. Math. 118 (1996), no. 2, 319-340. MR.1385280 (97f:52015)

Department of Mathematics, University of Missouri, Columbia, Missouri 65211

E-mail address: koldobskiya@missouri.edu 